\title{
Reducing Malignant Ascites and Long-Term Survival in a Patient with Recurrent Gastric Cancer Treated with a Combination of Docetaxel and Mistletoe Extract
}

\author{
Sung Jin Oh \\ Department of Surgery, Haeundae Paik Hospital, Inje University College of Medicine, \\ Busan, Republic of Korea
}

\section{Keywords}

Advanced gastric cancer · Docetaxel · Mistletoe

\begin{abstract}
Recurrent gastric cancer with peritoneal seeding is associated with poor overall survival. A 71-year-old man with advanced gastric cancer underwent radical total gastrectomy (stage IIlb, T4b N1 M0). Abdomino-pelvic computed tomography performed 7 months after surgery revealed increased ascites with enhanced peritoneal thickening. He was diagnosed with peritoneal seeding and received second-line chemotherapy (irinotecan). However, he refused chemotherapy due to the side effects (general weakness, nausea, and vomiting) after 1 cycle. He started receiving alternative therapy with Viscum album extract (VAE) (mistletoe) instead of chemotherapy. Malignant ascites gradually decreased after mistletoe therapy. So we started third-line chemotherapy (docetaxel) with VAE. The patient achieved good health with complete response postoperatively 32 months after combination treatment and survived over 60 months after surgery without disease progression. We report a case of recurrent gastric cancer that was successfully treated with a combination of docetaxel monotherapy and long-term mistletoe extract treatment.

\section{Introduction}

Stomach cancer is the fifth most diagnosed malignant tumor and the third most common cause of malignant tumor-related death [1]. Although, the survival rate of gastric cancer has increased owing to skillful D2 lymphadenectomy and development of an anticancer drug, the prognosis of recurrent gastric cancer remains poor. After curative resection (R0) for gastric 

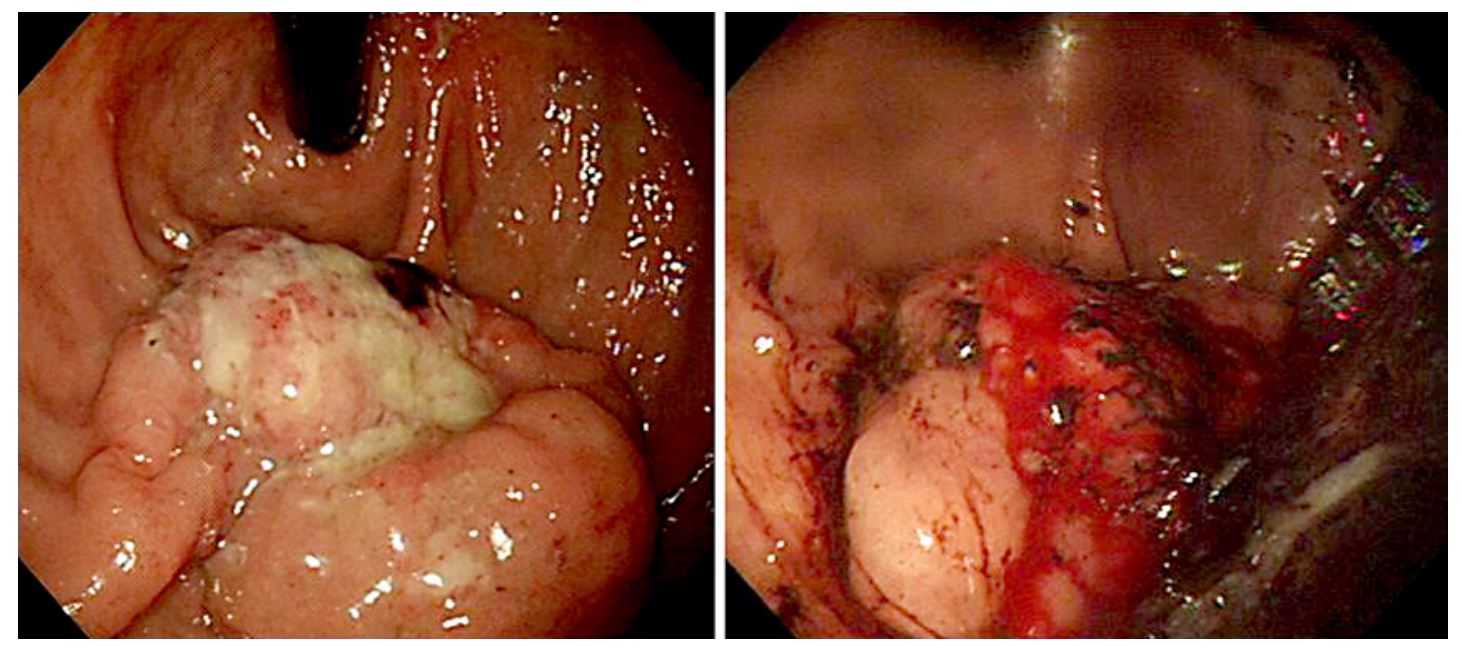

Fig. 1. Endoscopic finding shows an approximately $2 \mathrm{~cm}$ sized lobulated lesion with normal mucosa at the anterior wall of the mid body.

cancer with invasion to adjacent organs (T4b), the peritoneum is one of the most common recurrent sites [2] and malignant ascites in peritoneal metastasis worsen the quality of life and lead to a poor prognosis. Additionally, second-line chemotherapy for the elderly with recurrent gastric cancer is not recommended owing to its side effects and a low compliance. However, several clinical trials have shown that Viscum album can improve the quality of life and reduce the side effects of chemotherapy. It also has synergistic cytotoxic and proliferation-inhibiting effects when used with chemotherapy [3, 4]. In Asian countries, a standard adjuvant regimen for gastric cancer is XELOX or S-1. However, there is no standard regimen for metastatic or recurrent gastric cancer. Docetaxel has been playing an important role as a new generation of anticancer drugs for treatment of metastatic or recurrent gastric cancer.

We herein report a case of an elderly patient with recurrent gastric cancer who was successfully treated with a combination of docetaxel monotherapy and long-term Viscum album extract (VAE).

\section{Case Presentation}

A 71-year-old man presented with hematemesis and hematochezia. He had hypertension, diabetes mellitus and chronic obstructive pulmonary disease. Initial esophagogastroduodenoscopy (EGD) revealed a fungating mass with central ulcer bleeding located in the greater curvature of the upper body. EGD of the biopsy specimens revealed the mass was an adenocarcinoma (Fig. 1). A contrast-enhanced stomach computerized tomography (CT) scan showed an endoluminal protruding mass with delayed enhancement and area of necrosis in the posterior wall of the gastric fundus and perigastric fat infiltration, and abutting to the spleen and retroperitoneum (Fig. 2). The initial carcinoembryonic antigen level was $4.6 \mathrm{ng} /$ $\mathrm{mL}$ and other tumor markers were within normal limits. In the field of operation, the gastric tumor appeared to invade the serosa and the spleen grossly and abutted to the pancreatic capsule. A curative intended total gastrectomy with D2 lymph node dissection and splenectomy was performed.

The Borrmann type III gastric cancer at the upper body of the greater curvature was $6.0 \times 4.5 \times 3.5 \mathrm{~cm}$ in size and histologically diagnosed as a moderately differentiated mucinous 

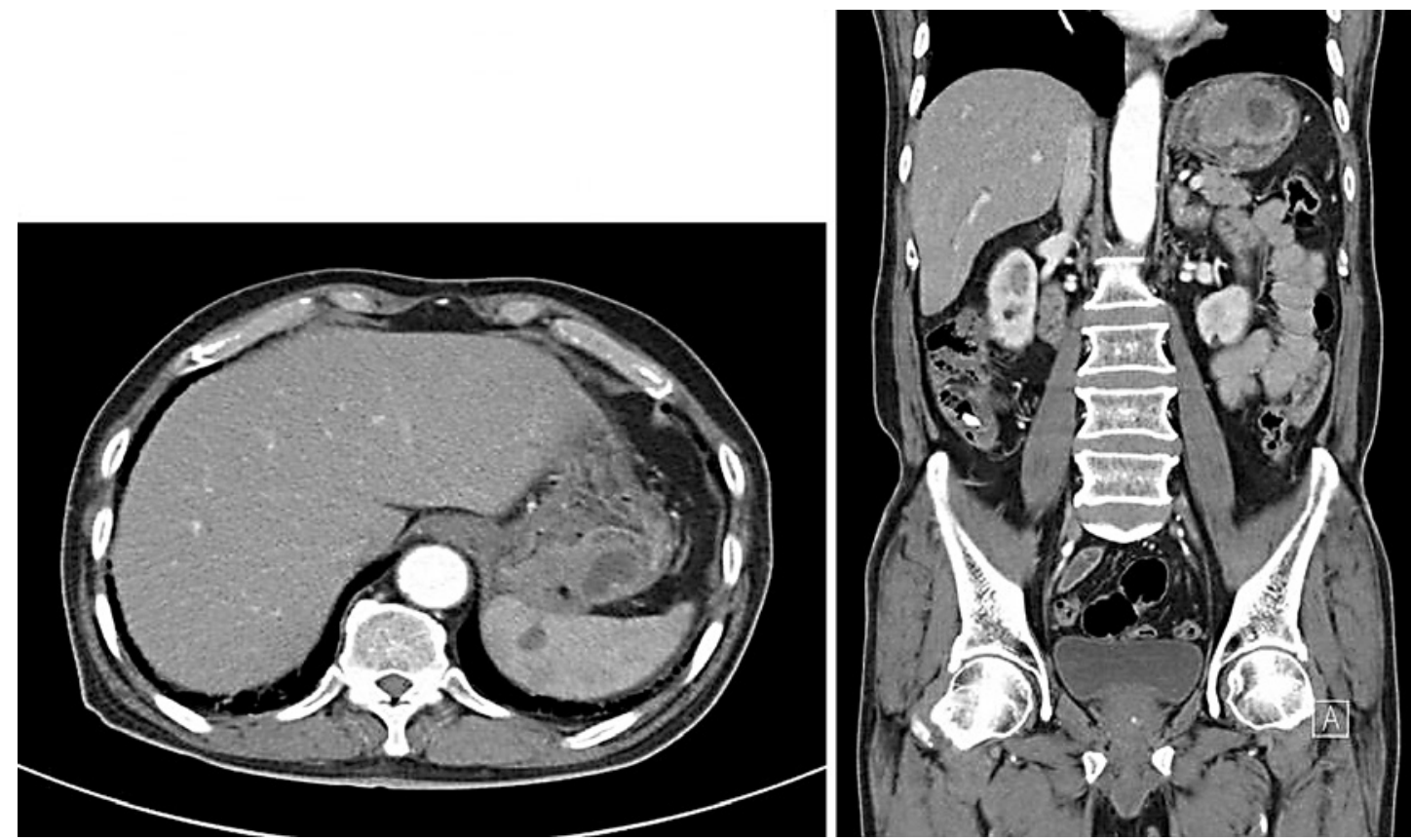

Fig. 2. Stomach computed tomography shows an approximately $5.6 \mathrm{~cm}$ sized endoluminal protruding mass with delayed enhancement and area of necrosis in the posterior wall of gastric fundus, which is perigastric fat infiltration, abutting to the spleen and ill-defined wedge-shaped low attenuated lesion in the spleen.

adenocarcinoma of the intestinal type. According to the seventh edition of the AJCC/UICC classification of gastric cancer, the case was classified as stage IIIb (T4b, N1, M0).

The patient's postoperative course was uneventful. The patient underwent adjuvant chemotherapy with the XELOX (capecitabine and oxaliplatin) regimen. After 8 courses of chemotherapy, the patient complained of general weakness and abdominal distension. Follow-up CT showed increased ascites with enhanced peritoneal thickening (Fig. 3a). The second-line chemotherapy regimen was changed to FOLFIRI (5-fluorouracil, leucovorin, irinotecan) regimen; however, the patient complained of generalized weakness, abdominal distension, and vomiting after finishing only one cycle and refused to receive further chemotherapy owing to side effects. After discussion and persuasion, the patient conditionally agreed to mistletoe (VAE) therapy rather than to intravenous chemotherapy. The general condition of the patient improved as malignant ascites gradually decreased after continuous administration of subcutaneous VAE three times a week. Therefore, we recommended the third-line chemotherapy as docetaxel monotherapy $(75 \mathrm{mg} / \mathrm{BSA})$ every 3 weeks with mistletoe (VAE) treatment. After 12 cycles, a follow-up CT scan performed 32 months postoperatively revealed complete response compared with the response revealed in the previous CT scan. The patient survived for 60 months after surgery without further disease progression (Fig. 3b).

\section{Discussion}

Even though the prognosis of recurrent or metastatic gastric cancer is very poor, active salvage therapy including chemotherapy, immunotherapy, and surgery for metastatic or recurrent gastric cancer might ensure long-term survival [5]. However, it is difficult to 
Fig. 3. a Abdomino-pelvic computed tomography reveals increased ascites with enhancing peritoneal thickening. b Abdomino-pelvic computed tomography shows nearly disappeared fluid collection in the abdominal cavity.
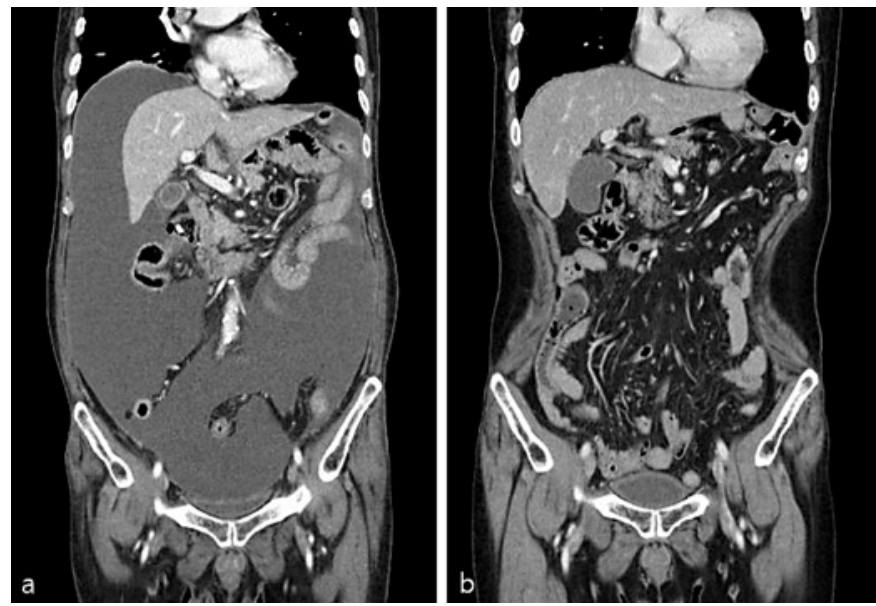

perform salvage therapy for elderly patients with recurrent gastric cancer who were immunocompromised due to the cytotoxicity of adjuvant chemotherapy. Therefore, it is essential to improve the quality of life and tolerability of chemotherapy for these patients.

Mistletoe extract treatment has been widely used for integrative cancer care, and Viscum album has cytotoxic effects against cancer cells and enhances the immune system in vivo and in vitro (activation of monocytes/macrophages, granulocytes, natural killer cells, $\mathrm{T}$ cells, dendritic cells, and induction of cytokines) $[3,6]$. Several clinical trials have reported that mistletoe extract treatment has shown an improvement in the survival time and quality of life of patients with cancer $[3,7]$, while clinical remissions of tumors have been reported only in small case reports $[8,9]$.

Even after curative resection among patients with $\mathrm{T} 4 \mathrm{~b}$ gastric cancer, the recurrence rate is high and one of the most common recurrence sites is the peritoneum [2]. Thus, peritoneal recurrence is often accompanied by malignant ascites, which has irritating problems in terms of abdominal distension, pain, restricted mobility, indigestion, and dyspnea. Repeated paracentesis for the control of malignant ascites may also cause bowel perforation, bacterial peritonitis, peritoneo-cutaneous fistula, and cellulitis [10]. Although there is no clear objective evidence of VAE effect on reducing the amount of malignant ascites, several studies showed that repeated intraperitoneal administration of VAE in patients with end-stage malignancies decreased the amount of malignant ascites. In addition, other studies showed that pleurodesis was induced by local intrapleural instillation of VAE in pleural cancer patients with malignant pleural effusion. Some researchers suggested that an effect of VAE is due to an activation of autoimmune reactions rather than mechanical sclerosis [11,12].

In Asian countries, XELOX or S-1 is a standard regimen as a postoperative adjuvant chemotherapy for advanced gastric cancer, but there is no standard chemotherapy regimen for recurrent or metastatic gastric cancer. A phase II trial showed that docetaxel monotherapy or combination therapy had considerable activity and were well tolerated in patients with an advanced gastric cancer [13]. As a single agent, docetaxel is generally well tolerated with patients with predominant toxicity of hematologic neutropenia. In addition, docetaxel-based combination regimens (5-fluorouracil and cisplatin, oxaliplatin, irinotecan, and biological agents) have been tried for the treatment of advanced, metastatic, and recurrent gastric cancer [14]. In our case, it was difficult to decide third-line chemotherapy regimen because the actual compliance with chemotherapy for this elderly patient was poor due to toxicities of chemotherapy and malignant ascites. We first performed percutaneous continuous drainage with an 8.5-French pigtail catheter to control the ascites under ultrasonographic 
Oh: Complete Remission of Peritoneal Metastases

guidance, but it had no effect. After administration of VAE for several months, the general condition of the patient was improved with reducing the amount of malignant ascites. Then we recommended the third-line chemotherapy as docetaxel monotherapy.

The patient, aged 71 years at diagnosis, had advanced gastric cancer with spleen invasion. After 8 courses of adjuvant chemotherapy, the patient complained of general weakness and malignant ascites due to peritoneal metastases. However, the disease was successfully controlled after the combination treatment using docetaxel monotherapy and mistletoe extract. We hope that the findings of this case will assist in the management of advanced-stage gastric cancer with peritoneal recurrence.

\section{Statement of Ethics}

Written informed consent for the publication of the patient's clinical details and images was obtained from the patient.

\section{Disclosure Statement}

The author has no conflicts of interest to declare.

\section{Funding Sources}

The author declared that this study has received no financial support.

\section{Author Contributions}

Sung Jin Oh drafted and revised the manuscript; Sung Jin Oh was involved in the conception and design of the case report.

\section{References}

1 Ferlay J, Soerjomataram I, Dikshit R, Eser S, Mathers C, Rebelo M, et al. Cancer incidence and mortality worldwide: sources, methods and major patterns in GLOBOCAN 2012. Int J Cancer. 2015;136(5):E359-86.

2 Zhu BY, Yuan SQ, Nie RC, Li SM, Yang LR, Duan JL, et al. Prognostic factors and recurrence patterns in T4 gastric cancer patients after curative resection. J Cancer. 2019;10(5):1181-8.

3 Kienle GS, Kiene H. Review article: influence of Viscum album L (European mistletoe) extracts on quality of life in cancer patients: a systematic review of controlled clinical studies. Integr Cancer Ther. 2010;9(2):14257.

4 Tröger W, Galun D, Reif M, Schumann A, Stanković N, Milićević M. Quality of life of patients with advanced pancreatic cancer during treatment with mistletoe: a randomized controlled trial. Dtsch Arztebl Int, 2014; 111(29-30):493-502.

5 Yoshida K, Yamaguchi K, Okumura N, Tanahashi T, Kodera Y. Is conversion therapy possible in stage IV gastric cancer: the proposal of new biological categories of classification. Gastric Cancer. 2016;19(2):329-38.

6 Siegle I, Fritz P, McClellan M, Gutzeit S, Mürdter TE. Combined cytotoxic action of Viscum album agglutinin-1 and anticancer agents against human A549 lung cancer cells. Anticancer Res. 2001;21(4a):2687-91.

7 Tröger W, Galun D, Reif M, Schumann A, Stanković N, Milićević M. Viscum album [L.] extract therapy in patients with locally advanced or metastatic pancreatic cancer: a randomised clinical trial on overall survival. Eur J Cancer. 2013;49(18):3788-97.

8 Werthmann PG, Kempenich R, Kienle GS. Erratum to: Long-term tumor-free survival in a patient with stage IV epithelial ovarian cancer undergoing high-dose chemotherapy and Viscum album extract treatment: a case report. Perm J. 2018;23:18-025. 
9 Hwang WY, Kang MH, Lee SK, Yeom JS, Jung MH. Prolonged stabilization of platinum-refractory ovarian cancer in a single patient undergoing long-term mistletoe extract treatment: case report. Medicine (Baltimore). 2019; 98(8):e14536.

10 Mackey JR, Venner PM. Malignant ascites: demographics, therapeutic efficacy and predictors of survival. Can J Oncol. 1996;6(2):474-80.

11 Bar-Sela G, Goldberg H, Beck D, Amit A, Kuten A. Reducing malignant ascites accumulation by repeated intraperitoneal administrations of a Viscum album extract. Anticancer Res. 2006;26(1b):709-13.

12 Stumpf C, Büssing A. Stimulation of antitumour immunity by intrapleural instillation of a Viscum album L. extract. Anticancer Drugs. 1997;8(Suppl 1):S23-6.

13 Ajani JA. Docetaxel for gastric and esophageal carcinomas. Oncology (Williston Park, NY). 2002;16(6 Suppl 6):89-6.

14 Nishiyama M, Wada S. Docetaxel: its role in current and future treatments for advanced gastric cancer. Gastric Cancer. 2009;12(3):132-41. 